\title{
Distribution of Lévy constants for quadratic numbers
}

\author{
by
}

\section{Christian Faivre (Marseille)}

0. Introduction. Let $x$ be an irrational number. We will denote by $\left[a_{0}(x), \ldots, a_{n}(x), \ldots\right]$ its regular continued fraction expansion and by $p_{n}(x) / q_{n}(x)=\left[a_{0}(x), \ldots, a_{n}(x)\right]$ the $n$th convergent. The famous Theorem of P. Lévy [12] states that for almost all $x \in \mathbb{R}$ (in the sense of Lebesgue) we have

$$
\lim _{n \rightarrow \infty} \frac{1}{n} \log q_{n}(x)=\frac{\pi^{2}}{12 \log 2} .
$$

One can prove that for a quadratic number $x$, the above sequence $n^{-1} \log q_{n}(x)$ is always convergent (see Section 2). Its limit is denoted here by $\beta(x)$ and called the Lévy constant of $x$. We also define the length of $x$ as $\varrho(x)=2 \log \varepsilon_{0}(x)$ (the terminology will be explained in the course of the paper) where $\varepsilon_{0}(x)=\frac{1}{2}\left(u_{0}+\sqrt{\Delta} v_{0}\right)$ is the fundamental solution of the Pell equation

$$
X^{2}-\Delta Y^{2}=4 .
$$

The number $\Delta$ is equal to $B^{2}-4 A C$ where $A x^{2}+B x+C=0$ is the minimal equation of $x$ in $\mathbb{Z}$, that is, $A>0, A, B, C \in \mathbb{Z}$ and $(A, B, C)=1$.

Our main results in this paper are:

\section{THEOREM I.}

$$
\sum_{\varrho(x) \leq X} \beta(x) \sim \frac{1}{4} e^{X} \quad(X \rightarrow+\infty) .
$$

THEOREM II.

$$
\sum_{\varrho(x) \leq X} 1 \sim \frac{3 \log 2}{\pi^{2}} e^{X} \quad(X \rightarrow+\infty) .
$$

We sum over the quadratic numbers in ]0,1[ purely periodic called reduced in this paper. We will show later that for every $X \geq 0$, the set of 
reduced quadratic numbers of length $\leq X$ is finite. Hence in Theorems I and II the summation is finite.

As a corollary of these theorems we obtain:

$$
\lim _{X \rightarrow+\infty} \frac{\sum_{\varrho(x) \leq X} \beta(x)}{\sum_{\varrho(x) \leq X} 1}=\frac{\pi^{2}}{12 \log 2},
$$

which corresponds to a conjecture of P. Liardet. This shows that in average, according to the length, the Lévy constants tend toward the "general" constant of P. Lévy's Theorem. The method of proof of Theorem II stems from works of Pollicott [14] and Mayer [13]. The first section of this paper deals with the length of a quadratic number. In the second we define for every quadratic number $x$ the Lévy constant of $x$ and give some of its properties. Finally, the third part of the paper is devoted to the proof of Theorems I and II.

Acknowledgement. I should like to thank P. Liardet for his advice during the preparation of this paper. I thank also the referee for his helpful remarks.

\section{Notations}

- If $x$ is a quadratic number, its conjugate will be denoted by $x^{*}$.

- A quadratic number $x \in] 0,1[$ is said to be reduced if its continued fraction expansion is such that $x=\left[0, \overline{a_{1}, \ldots, a_{N}}\right]$.

- $T:[0,1] \longmapsto[0,1[$ is the transformation of continued fractions, that is, $T(0)=0$ and $T(x)=1 / x-[1 / x]$ for $x \neq 0$. It is well-known that $T$ preserves Gauss's measure $d \mu=d x / \log 2(1+x)$ and that $T$ is ergodic with respect to this measure [1].

- We identify the linear fractional transformation $g(z)=(a z+b)(c z+d)^{-1}$ where $a, b, c, d \in \mathbb{R}$ and $a d-b c=1$ with the matrix

$$
A=\left(\begin{array}{ll}
a & b \\
c & d
\end{array}\right) \in \operatorname{PSL}(2, \mathbb{R}),
$$

and we denote by $R(g)$ the spectral radius of $A$. The transformation $g$ is said to be hyperbolic if $|a+d|>2$ (it is equivalent to say that there are two fixed points in $\mathbb{R} \cup\{\infty\}$ ).

- An element $f \in \operatorname{PSL}(2, \mathbb{Z})$ is said to be primitive if $f=h^{n}$ (with $h \in \operatorname{PSL}(2, \mathbb{Z})$ and $n \geq 1)$ implies that $n=1$.

- $\mathrm{GL}(2, \mathbb{Z})$ acts on the set of quadratic numbers by

$$
P x=\frac{a x+b}{c x+d}, \quad P=\left(\begin{array}{ll}
a & b \\
c & d
\end{array}\right) .
$$

Two quadratic numbers in the same orbit are said to be $\operatorname{GL}(2, \mathbb{Z})$-equivalent. If we can choose $P$ in $\operatorname{SL}(2, \mathbb{Z})$ we will say simply that they are equivalent. 
1. The length of a quadratic number. In the introduction we have defined for every quadratic number $x$ the length of $x$ denoted by $\varrho(x)$. In this section we shall justify the use of the word length.

Let $H=\{(x, y) ; y>0\}$ be the hyperbolic plane with its classical complete metric $d s^{2}=y^{-2}\left(d x^{2}+d y^{2}\right)$. For this metric the curvature of $H$ is constant and equal to -1 . Elements of $\operatorname{PSL}(2, \mathbb{R})$ are isometries of $H$. The geodesics $\gamma: \mathbb{R} \rightarrow H$ for the hyperbolic metric are supported by vertical halflines and the half-circles centered on the real axis. Let $M=H / \operatorname{PSL}(2, \mathbb{Z})$ be the modular surface. It is a Riemann surface, the geodesics of $M$ are by definition the $p \circ \gamma$ where $\gamma: \mathbb{R} \rightarrow H$ is a geodesic of $H$ and $p: H \rightarrow M$ the canonical projection. The following theorem is known (see [16]) and gives the closed geodesics (i.e. periodic geodesics) on the modular surface. For the reader's convenience we will give a complete proof of this theorem.

THEOREM 1.1. (i) Let $\gamma$ be a geodesic of $H$ joining a quadratic number $x$ and its conjugate $x^{*}$. Then $p \circ \gamma$ is a closed geodesic of $M$ and all the closed geodesics on $M$ arise in this way.

(ii) The length of $p \circ \gamma$ is given by $\varrho(x)=2 \log \varepsilon_{0}(x)$.

R e m a r k. The assertion (ii) justifies the use of the word length for $\varrho(x)$. For the proof of Theorem 1.1 we will need the following lemmas.

Lemma 1.1 [11]. Let $x$ be a quadratic number. Then $\{g \in \operatorname{PSL}(2, \mathbb{Z})$; $g(x)=x\}$ is an infinite cyclic subgroup of $\operatorname{PSL}(2, \mathbb{Z})$ and a generator is

$$
U(x)=\left(\begin{array}{cc}
\frac{u_{0}-B v_{0}}{2} & -C v_{0} \\
A v_{0} & \frac{u_{0}+B v_{0}}{2}
\end{array}\right) .
$$

It will be noticed that the spectral radius of $U(x)$ is precisely $\varepsilon_{0}(x)$. The quantities $A, B, C, u_{0}, v_{0}$ and $\varepsilon_{0}(x)$ have been defined in the introduction.

Lemma 1.2. Let $\gamma_{1}, \gamma_{2}: \mathbb{R} \rightarrow H$ be two geodesics such that $p \circ \gamma_{1}=p \circ \gamma_{2}$. Then there exists a $g \in \operatorname{PSL}(2, \mathbb{Z})$ such that $g \circ \gamma_{1}=\gamma_{2}$.

Proof. This lemma is not entirely obvious since $p$ is not a covering. Since $\operatorname{PSL}(2, \mathbb{Z})$ is countable and its every element (different from the identity) has at most one fixed point in $H$, there exists $t_{0} \in \mathbb{R}$ such that $\gamma_{1}\left(t_{0}\right)$ is fixed by no element of $\operatorname{PSL}(2, \mathbb{Z})$ different from the identity. Let $g \in \operatorname{PSL}(2, \mathbb{Z})$ such that $\gamma_{2}\left(t_{0}\right)=g \circ \gamma_{1}\left(t_{0}\right)$. As every Fuchsian group, $\operatorname{PSL}(2, \mathbb{Z})$ acts on $H$ properly and discontinuously [17]. Hence from the choice of $\gamma_{1}\left(t_{0}\right)$ there is an open set $U \subset H$ such that $\gamma_{1}\left(t_{0}\right) \in U$ and $g(U) \cap U=\emptyset$ for every $g \in \operatorname{PSL}(2, \mathbb{Z})$ with $g \neq I$. Consider $\varepsilon>0$ such that $\gamma_{1}(] t_{0}-\varepsilon, t_{0}+\varepsilon[) \subset U$ and $\gamma_{2}(] t_{0}-\varepsilon, t_{0}+\varepsilon[) \subset g(U)$. For all $t_{0}-\varepsilon<t<t_{0}+\varepsilon$ we can write $\gamma_{2}(t)=g_{t} \circ \gamma_{1}(t)$ with $g_{t} \in \operatorname{PSL}(2, \mathbb{Z})$. Thus $\gamma_{2}(t) \in g_{t}(U) \cap g(U)$ and $g_{t}=g$ from the choice of $U$. The two geodesics $\gamma_{2}$ and $g \circ \gamma_{1}$ coincide 
on the open interval $] t_{0}-\varepsilon, t_{0}+\varepsilon\left[\right.$ and consequently $\gamma_{2}(t)=g \circ \gamma_{1}(t)$ for all $t \in \mathbb{R}$.

LEMMA 1.3. Let $g(z)=(a z+b)(c z+d)^{-1}$ be a hyperbolic transformation from $\operatorname{PSL}(2, \mathbb{Z})$ and $x, x^{*}$ its two fixed points. For all $z \in H$ in the half circle of $H$ having $x$ and $x^{*}$ as endpoints, we have $d(z, g(z))=2 \log R(g)$.

Proof. Use the expression of the hyperbolic distance with the crossratio.

Proof of Theorem 1.1. We may suppose $\left\|\gamma^{\prime}(t)\right\|=1$ relative to the hyperbolic metric. Let $g \neq I$ be an element of $\operatorname{PSL}(2, \mathbb{Z})$ such that $g(x)=x$ (thus $g\left(x^{*}\right)=x^{*}$ ) (Lemma 1.1). From Lemma 1.3 we have $g \circ \gamma(t)=\gamma(t \pm 2 \log R(g))$, hence $p \circ \gamma$ is periodic and $2 \log R(g)$ is a period. Let now $L>0$ be another period of $p \circ \gamma$ and $h$ a generator of $\{g \in \operatorname{PSL}(2, \mathbb{Z}) ; g(x)=x\}$. From the above $2 \log R(h)$ is also a period and there exists $k \in \operatorname{PSL}(2, \mathbb{Z}), k \neq I$ such that $k \circ \gamma(t)=\gamma(t+L)$ (Lemma 1.2). By letting $t \rightarrow+\infty$ or $-\infty$ we notice that $k(x)=x$, thus $k=h^{n}$ for some $n \in \mathbb{Z}-\{0\}$. We deduce $k \circ \gamma(t)=\gamma(t \pm 2 n \log R(h))=\gamma(t+L)$, thus $L=2|n| \log R(h)$ since $\gamma$ is injective. Hence $2 \log R(h)$ is the smallest period $>0$ of $p \circ \gamma$. Since $\left\|\gamma^{\prime}(t)\right\|=1$, the length of $p \circ \gamma$ is precisely $2 \log R(h)$, and (ii) follows from Lemma 1.1.

It remains to prove the last assertion of (i). Let $p \circ \gamma$ be a closed geodesic with period $L \neq 0$. By Lemma 1.2, there exists $g \in \operatorname{PSL}(2, \mathbb{Z}), g \neq I$, such that $g \circ \gamma(t)=\gamma(t+L)$. By letting $t \rightarrow+\infty$ and $-\infty$ we have $g(y)=y$ and $g(z)=z$ where $y, z(y \neq z)$ are the endpoints of $\gamma$. This proves that $y, z$ are conjugate quadratic numbers and completes the proof of the theorem.

2. The Lévy constant of a quadratic number. Let $x \in] 0,1[$ be an irrational number. The following formula is fundamental in continued fraction theory:

$$
x=\frac{p_{n-1} T^{n} x+p_{n}}{q_{n-1} T^{n} x+q_{n}} \quad(n \geq 0) .
$$

As in the introduction, $p_{n} / q_{n}$ is the $n$th convergent of $x$ and we put as usual $p_{-1}=1, q_{-1}=0$ and $p_{0}=0, q_{0}=1$. From (1) we obtain immediately

$$
T^{n} x=-\frac{x q_{n}-p_{n}}{x q_{n-1}-p_{n-1}} .
$$

This gives the useful lemma:

Lemma 2.1. For all $n \geq 0, x T x \ldots T^{n} x=(-1)^{n}\left(x q_{n}-p_{n}\right)$.

Applying again (1), we deduce for $n \geq 0$

$$
q_{n}\left|x q_{n-1}-p_{n-1}\right|=\frac{q_{n}}{q_{n-1} T^{n} x+q_{n}} .
$$


So

$$
1 / 2 \leq q_{n}\left|x q_{n-1}-p_{n-1}\right| \leq 1 .
$$

Thus Lemma 2.1 and Birkhoff's ergodic theorem show that for almost all $x \in[0,1]$

$$
\lim _{n \rightarrow \infty} \frac{1}{n} \log \left|x q_{n-1}-p_{n-1}\right|=\int_{0}^{1} \log x d \mu(x)=\frac{-\pi^{2}}{12 \log 2} .
$$

From the inequalities (2) we obtain the well-known Theorem of P. Lévy.

Theorem 2.1 [12]. For almost all $x \in \mathbb{R}$

$$
\lim _{n \rightarrow \infty} \frac{\log q_{n}(x)}{n}=\frac{\pi^{2}}{12 \log 2} .
$$

From previous arguments, we notice that for an irrational number $x \in$ ] 0,1 , the sequence $n^{-1} \log q_{n}(x)$ is convergent if and only if $n^{-1} \sum_{i=0}^{n-1} \log T^{i} x$ is convergent and in this case the two limits are opposite. From this, for a reduced quadratic number $x=\left[0, \overline{a_{1}, \ldots, a_{N}}\right]$ we will have

$$
\lim _{n \rightarrow \infty} \frac{1}{n} \log q_{n}(x)=\frac{-1}{N} \sum_{i=0}^{N-1} \log T^{i} x .
$$

Furthermore if $y, z$ are two $\operatorname{GL}(2, \mathbb{Z})$-equivalent quadratic numbers, there exist integers $a, b, j$ with $(a, b) \neq(0,0)$ such that $q_{n+j}(y)=a p_{n}(z)+b q_{n}(z)$ for all sufficiently large $n$. Thus $n^{-1} \log q_{n}(y)-n^{-1} \log q_{n}(z)=o(1)$. As every quadratic number is $\mathrm{GL}(2, \mathbb{Z})$-equivalent to a reduced one we get:

Proposition 2.1. (i) For every quadratic number $x$, the sequence $n^{-1} \log q_{n}(x)$ is convergent. Its limit is denoted by $\beta(x)$ and called the Lévy constant of $x$.

(ii) If $x=\left[0, \overline{a_{1}, \ldots, a_{N}}\right]$ then $\beta(x)=-N^{-1} \sum_{i=0}^{N-1} \log T^{i} x$.

(iii) If $x, y$ are two $\mathrm{GL}(2, \mathbb{Z})$-equivalent quadratic numbers, then $\beta(x)=$ $\beta(y)$.

(iv) For every quadratic number $x$ one has $\beta(x)=\beta\left(x^{*}\right)$.

Remark. (i) and (ii) are results of H. Jager and P. Liardet [9]. The previous arguments give a new proof of these.

Proof. The only point which remains to prove is (iv). We can suppose that $x$ is reduced, $x=\left[0, \overline{a_{1}, \ldots, a_{N}}\right]$. From Galois's Theorem [4], $-1 / x^{*}=$ $\left[0, \overline{a_{N}, \ldots, a_{1}}\right]$. We put $y=-1 / x^{*}$ and write $y=\left[0, b_{1}, b_{2}, \ldots\right]$. By a classical result

$$
\frac{q_{n-1}(y)}{q_{n}(y)}=\left[0, b_{n}, \ldots, b_{1}\right] \quad(n \geq 1) .
$$


Writing $v_{n}=\left[0, b_{n}, \ldots, b_{1}\right]$ we have for all $1 \leq r \leq N$ and $k \geq 1$

$$
v_{N k+r}=\left[0, a_{N-r+1}, \ldots, a_{N}, a_{1} \ldots a_{N}, \ldots, a_{1} \ldots a_{N}\right],
$$

with $k$ blocks $a_{1} \ldots a_{N}$. Hence the sequence $v_{N k+r}$ converges to $T^{N-r}(x)$ as $k \rightarrow \infty$. Thus

$$
\lim _{n \rightarrow \infty} \frac{1}{n} \log q_{n}(y)=\lim _{n \rightarrow \infty} \frac{-1}{n} \sum_{i=1}^{n} \log v_{i}=\frac{-1}{N} \sum_{i=0}^{N-1} \log T^{i} x=\beta(x) .
$$

From (iii) it follows that $\beta(y)=\beta\left(x^{*}\right)$ and this completes the proof.

Proposition 2.2. Let $x$ be a reduced quadratic number, $x=$ $\left[0, \overline{a_{1}, \ldots, a_{N}}\right]$, where $N$ is the minimal period length. Then the following assertions hold:

(i) $\varrho(x)=2 \log \varepsilon_{0}(x)=-2 \alpha_{N} \sum_{i=0}^{N-1} \log T^{i} x$ with $\alpha_{N}=1$ if $N$ is even, 2 otherwise.

(ii) $\beta(x)=-N^{-1} \sum_{i=0}^{N-1} \log T^{i} x$.

Proof. (ii) is already known. The assertion (i) follows from the following formula known as Smith's formula [19] (see also [3] for another proof):

$$
\varepsilon_{0}(x)= \begin{cases}\left(x T x \ldots T^{N-1} x\right)^{-1} & \text { if } N \text { is even } \\ \left(x T x \ldots T^{N-1} x\right)^{-2} & \text { if } N \text { is odd. }\end{cases}
$$

Let $x$ be a reduced quadratic number and let $t \mapsto \gamma(t)$ be a geodesic in $H$ parametrized by arc length joining $x$ to $x^{*}(<-1)$ and oriented from $x$ to $x^{*}$. Such a geodesic will be called associated with $x$. All other geodesics that have this property are of the form $t \mapsto \gamma(t+k)$ with $k \in \mathbb{R}$. There exists a unique primitive hyperbolic transformation $g$ such that

$$
\gamma(t+2 \log R(g))=g(\gamma(t)) \quad(t \in \mathbb{R}) .
$$

More precisely, $g$ is one of the two primitive transformations leaving $x$ fixed. We will say that $g$ is the transformation associated with $x$. If $y$ is another reduced quadratic number with $y=h(x)(h \in \operatorname{PSL}(2, \mathbb{Z}))$ then the transformation associated with $y$ is $h g h^{-1}$. Hence we have a map between the classes of equivalent reduced quadratic numbers and the conjugacy classes of hyperbolic primitive transformations. We denote it by $\Lambda$.

Proposition 2.3. The map $\Lambda$ is bijective. To the class determined by a reduced quadratic number $x$ corresponds a conjugacy class $\langle g\rangle$ such that $\varrho(x)=2 \log R(g)$.

Proof. Consider $x, y$ whose associated transformations are precisely $g$ and $h g h^{-1}$. Let $\gamma_{1}$ (resp. $\gamma_{2}$ ) be a geodesic of $H$ associated with $x$ (resp. $y$ ). We put $L=2 \log R(g)$. We have $\gamma_{1}(t+L)=g \gamma_{1}(t)$ and $\gamma_{2}(t+L)=$ $h g h^{-1} \gamma_{2}(t)$. By letting $t \rightarrow-\infty$, the second equality yields $g\left(h^{-1} y\right)=h^{-1} y$. Thus $h^{-1} y=x$ or $x^{*}$. Suppose that $h^{-1} y=x^{*}$ and put $\gamma_{3}(t)=h^{-1} \gamma_{2}(-t)$. 
We have $\gamma_{3}(t+L)=g^{-1} \gamma_{3}(t)$ and $\gamma_{3}$ is associated with $x$; this implies $g=g^{-1}$, hence $g^{2}=I$, which is impossible since $g$ is hyperbolic. Hence $\Lambda$ is injective.

To prove the surjectivity of $\Lambda$, let $\langle g\rangle$ be a conjugacy class of primitive hyperbolic transformations. We can suppose that one of the fixed points of $g$ is a reduced quadratic number, say $x$. Let $\gamma$ be a geodesic associated with $x$. We have for all $t \in \mathbb{R}$, either $\gamma(t+2 \log R(g))=g \gamma(t)$ or $\gamma(t+2 \log R(g))=$ $g^{-1} \gamma(t)$. If the first equality occurs the proof is finished. If the second does, let $y$ be a reduced quadratic number equivalent to $x^{*}, y=h\left(x^{*}\right)$ (where $h \in \operatorname{PSL}(2, \mathbb{Z}))$. We put $\gamma_{1}(t)=h \gamma(-t)$; then $\gamma_{1}$ is associated with $y$ and $\gamma_{1}(t+2 \log R(g))=h g h^{-1} \gamma_{1}(t)$, thus $\Lambda$ is onto.

3. Proofs of Theorems I and II. First we introduce some definitions. For a quadratic number $x$ we denote by $\operatorname{per}(x)$ the least period length in its continued fraction expansion. It is convenient to define also the length of a finite word $m=a_{1} \ldots a_{N}$ built on the alphabet $\mathbb{N}-\{0\}$ as $\varrho(m)=-2 \alpha_{N} \sum_{i=0}^{N-1} \log T^{i} x$ where $x=\left[0, \overline{a_{1}, \ldots, a_{N}}\right]$. Notice that we do not necessarily have $\operatorname{per}(x)=N$. We also put $|m|=N$ for short. From now on $m$ denotes a word. We show that there exist a finite number of words (and therefore of reduced quadratic numbers) of length $\leq X$.

Proposition 3.1. For all $X \geq 0$ the set of words $m$ such that $\varrho(m) \leq X$ is finite.

Proof. Let $m=a_{1} \ldots a_{N}$ be a word with $\varrho(m) \leq X$ and put $x=$ $\left[0, \overline{a_{1}, \ldots, a_{N}}\right]$. We have

$$
T^{i} x=\left[0, a_{i+1}, a_{i+2}+T^{i+2} x\right]<\left[0, a_{i+1}, a_{i+2}+1\right],
$$

hence

$$
\varrho(m)>\sum_{i=0}^{N-1} \log \left(a_{i+1}+\frac{1}{a_{i+2}+1}\right),
$$

so that $1 \leq a_{i}<e^{X}$ for $i=1,2, \ldots, N$ and $N \log \left(1+\left(e^{X}+1\right)^{-1}\right)<X$, which proves the proposition.

Now we introduce the following functions:

$$
\begin{gathered}
\pi_{0}(X)=\sum_{\varrho(x) \leq X} 1, \quad \pi_{1}(X)=\sum_{\varrho(m) \leq X} 1, \quad \pi_{2}(X)=\sum_{\substack{\varrho(x) \leq X \\
\operatorname{per}(x) \text { odd }}} 1, \\
\pi(X)=\sum_{2 \log R(g) \leq X} 1, \quad \theta(X)=\sum_{2 \log R(g) \leq X} 2 \log R(g) .
\end{gathered}
$$

Let us remember that for $\pi_{0}(X)$ the sum concerns all the reduced quadratic numbers whose length is $\leq X$. For $\pi(X)$ the sum concerns all the conjugacy 
classes of primitive hyperbolic transformations of $\operatorname{PSL}(2, \mathbb{Z})$. We recall that $R(g)$ means the spectral radius of $g$. The behaviour of $\pi(X)$ as $X \rightarrow \infty$ is given by the following theorem.

Theorem 3.1. $\pi(X) \sim e^{X} / X(X \rightarrow+\infty)$.

For a proof using Selberg's trace formula see [8] (Theorem 3.5, p. 475). For another proof see [10].

Proposition 3.2. $\theta(X) \sim e^{X}(X \rightarrow+\infty)$.

Proof. Let $a=a(X)<X$ be a function which will be specified later. We have

$$
\begin{gathered}
\theta(X) \geq \sum_{a<2 \log R(g) \leq X} 2 \log R(g) \geq a(\pi(X)-\pi(a)), \\
\frac{\theta(X)}{e^{X}} \geq \frac{a}{X} \frac{X \pi(X)}{e^{X}}-\frac{a \pi(a)}{e^{a}} e^{a-X} .
\end{gathered}
$$

By taking $a=X-\log X$, we have from Theorem 3.1

$$
\liminf _{X \rightarrow+\infty} \theta(X) e^{-X} \geq 1 \text {. }
$$

On the other hand, from the inequality $\theta(X) \leq X \pi(X)$ we deduce

$$
\limsup _{X \rightarrow+\infty} \theta(X) e^{-X} \leq 1
$$

which completes the proof.

Theorem 3.2. $\sum_{\varrho(x) \leq X} \beta(x) \sim \frac{1}{4} e^{X}(X \rightarrow+\infty)$.

Proof. Let $x=\left[0, \overline{a_{1}, \ldots, a_{N}}\right]$ be a reduced quadratic number with $N=\operatorname{per}(x)$. Notice that

(i) If $N$ is odd then $x$ and $T x$ are equivalent.

(ii) If $N$ is even, $x$ and $T x$ are not equivalent.

For (i) it is enough to remark that $T x=\left[0, a_{2}, \ldots, a_{N}+x\right]=T_{a_{2}} \circ$ $\ldots \circ T_{a_{N}}(x)$ where $T_{a}(x)=(x+a)^{-1}$. The assertion (ii) follows from the following result (see [7]): $\left[b_{0}, b_{1}, \ldots\right]$ and $\left[c_{0}, c_{1}, \ldots\right]$ are equivalent if and only if there exist $p, q \in \mathbb{N}$ of the same parity such that $b_{p+i}=c_{q+i}$ for all $i \geq 0$. The set of reduced quadratic numbers equivalent to $x$ will be denoted by $\bar{x}$. We have $\bar{x} \subset\left\{x, T x, \ldots, T^{N-1} x\right\}$ and furthermore every $T^{i} x$ for $i \geq 0$ is equivalent to $x$ or $T x$. Thus if $N$ is odd card $\bar{x}=N$, otherwise $\operatorname{card} \bar{x}=N / 2$. Since $\varrho\left(T^{i} x\right)=\varrho(x)$ for all $i \geq 0$ we may define the length of the class $\bar{x}$. Now consider the function

$$
F(X)=\sum_{\varrho(\tau) \leq X} \varrho(\tau)
$$


where $\tau$ runs through the set of classes. From Proposition 2.2 we deduce

$$
F(X)=\sum_{N=1}^{\infty} \frac{\alpha_{N}^{\prime}}{N} \sum_{\begin{array}{c}
x \operatorname{reduced} \\
\operatorname{per}(x)=N \\
\varrho(x) \leq X
\end{array}}-2 \alpha_{N} \sum_{i=0}^{N-1} \log T^{i} x,
$$

where $\alpha_{N}^{\prime}=1$ if $N$ is odd, 2 otherwise. Hence $F(X)=4 \sum_{\varrho(x) \leq X} \beta(x)$. From Proposition 2.3, we have $F(X)=\theta(X)$, and the proof follows from Proposition 3.2.

Corollary 3.1. $\pi_{0}(X)=O\left(e^{X}\right)$.

Proof. For all quadratic numbers $x$, we have the inequality $\beta(x) \geq$ $\log G$ where $G=\frac{1}{2}(\sqrt{5}+1)$. Indeed, $q_{n}(x) \geq q_{n}^{\prime}$ with $q_{0}^{\prime}=q_{1}^{\prime}=1$ and $q_{n+2}^{\prime}=q_{n+1}^{\prime}+q_{n}^{\prime}$ for $n \geq 0$. Since the sequence $n^{-1} \log q_{n}^{\prime}$ converges to $\log G$ the corollary is proved.

Proposition 3.3. $\pi_{1}(X)=\pi_{0}(X)+\pi_{2}(X)+o\left(e^{X}\right)$. In particular, $\pi_{1}(X)=O\left(e^{X}\right)$.

Proof. For $k \geq 1$, we put

$$
\Xi_{k}(X)=\pi_{0}\left(\frac{X}{k}\right)+\pi_{0}\left(\frac{X}{k+1}\right)+\ldots
$$

We have $\pi_{0}(X / r)=0$ if $r \sigma>X$ where $\sigma$ denotes the smallest length for quadratic numbers. It can be seen that $\sigma=4 \log G$ where $G=\frac{1}{2}(\sqrt{5}+1)$. From $\pi_{0}(X)=O\left(e^{X}\right)$ we deduce easily $\Xi_{k}(X)=O\left(e^{X / k}\right)$. We have

$$
\begin{aligned}
\sum_{\varrho(m) \leq X} 1 & =\sum_{\substack{\left(\alpha_{N} / \alpha_{p}\right) k \cdot \varrho(x) \leq X \\
k \geq 1 ; p=\operatorname{per}(x) \\
N=k \cdot p}} 1 \\
& =\pi_{0}(X)+\sum_{\substack{\varrho(x) \leq X \\
p \text { odd }}} 1+\sum_{\substack{2 \varrho(x) \leq X \\
p \text { even }}} 1+\sum_{\substack{\left(\alpha_{N} / \alpha_{p}\right) k \cdot \varrho(x) \leq X \\
k \geq 3}} 1 .
\end{aligned}
$$

In the second and the last sum the summation is extended over the couples $(k, x)$ with $k \geq 1$. Since the last two sums are respectively dominated by $\pi_{0}(X / 2)=O\left(e^{X / 2}\right)$ and $\Xi_{3}(2 X)=O\left(e^{2 X / 3}\right)$ the proposition is proved.

For $\operatorname{Re}(s)>1$, we define

$$
F(s)=\int_{0}^{\infty} e^{-s t} d \pi_{1}(t)=\sum_{m} e^{-s \varrho(m)} .
$$

Since $\pi_{1}(X)=O\left(e^{X}\right)$ (Proposition 3.3), the integral and the series are absolutely convergent and $F$ is holomorphic for $\operatorname{Re}(s)>1$. By definition of 
the length of a word we have another expression for $F$ :

$$
F(s)=\sum_{n=1}^{\infty} \sum_{a_{1}, \ldots, a_{n}}\left(x T x \ldots T^{n-1} x\right)^{2 \alpha_{n} s} .
$$

The second sum is extended over all $a_{1}, \ldots, a_{n} \in \mathbb{N}-\{0\}$ and $x=x_{a_{1}, \ldots, a_{n}}=$ $\left[0, \overline{a_{1}, \ldots, a_{n}}\right]$ for short. We will show that $F$ extends meromorphically to the half plane $\operatorname{Re}(s)>\frac{1}{2}$ with only one pole on the line $\operatorname{Re}(s)=1$ which is simple, located at $s=1$, with a residue equal to $(3 \log 2) / \pi^{2}$. The proof uses functional analysis and especially Fredholm's theory. Let $1<a<b<3 / 2$. For $\lambda>0$, we write $D_{\lambda}=\{z ;|z-1| \leq \lambda\}$. Let $E$ be the Banach space of functions continuous on $D_{a}$ and holomorphic in the interior. $E$ is endowed with the supremum norm. If $v$ is a Fredholm kernel, we denote as usual by $\|v\|_{1}$ its trace norm and by $\widetilde{v}$ its associated operator. We refer to [6] and [5] for Fredholm's theory. For $\operatorname{Re}(s)>\frac{1}{2}$, we consider the transfer operator of $E$ given by

$$
L(s)=\sum_{n=1}^{\infty} L_{n}(s)
$$

where

$$
L_{n}(s)(f)(z)=f\left(\frac{1}{z+n}\right)\left(\frac{1}{z+n}\right)^{2 s} \quad(n \geq 1) .
$$

Remark 1. We write $T_{n}(z)=(z+n)^{-1}$. For all $n \geq 1$ we see easily that $T_{n}\left(D_{3 / 2}\right)$ is contained in $\{z ; z \neq 0,|z-1| \leq 1\}$.

In the definition of $L_{n}$, we choose the principal branch of the logarithm. We also define, for $\operatorname{Re}(s)>\frac{1}{2}$, the Fredholm kernels

$$
v_{n}(s)=\sum_{j=0}^{\infty} \lambda_{n, j}(s) \otimes e_{j}, \quad v(s)=\sum_{n=1}^{\infty} v_{n}(s),
$$

where $e_{j} \in E$ is given by $e_{j}(z)=(z-1)^{j}$ and $\lambda_{n, j} \in E^{*}$ by $\lambda_{n, j}(s)(f)=$ $(j !)^{-1} g_{n}^{(j)}(1)$ with $g_{n}(z)=f\left(T_{n}(z)\right)\left(T_{n}(z)\right)^{2 s}$. Let us show that $v_{n}(s)$ and $v(s)$ are well defined. From Remark 1, the function $g_{n}$ is holomorphic in $D_{3 / 2}$. Hence, from Cauchy's inequalities

$$
\left|\lambda_{n, j}(s)(f)\right| \leq \frac{1}{b^{j}} \operatorname{Max}_{|z-1|=b}\left|g_{n}(z)\right| \quad(f \in E) .
$$

But for all $z \in D_{3 / 2}$,

$$
\left|g_{n}(z)\right| \leq\|f\|_{\infty} \frac{e^{-\operatorname{Im}(2 s) \operatorname{Arg}(1 /(z+n))}}{|z+n|^{2 \sigma}} \leq\|f\|_{\infty} \frac{e^{\pi|\operatorname{Im}(2 s)|}}{\left(n-\frac{1}{2}\right)^{2 \sigma}} .
$$


Hence by setting $k(s)=e^{\pi|\operatorname{Im}(2 s)|}$,

$$
\begin{aligned}
\left\|\lambda_{n, j}(s)\right\| & \leq \frac{k(s)}{b^{j}\left(n-\frac{1}{2}\right)^{2 \sigma}}, \\
\sum_{j=0}^{\infty}\left\|\lambda_{n, j}(s)\right\|\left\|e_{j}\right\| & \leq \frac{k(s)}{\left(n-\frac{1}{2}\right)^{2 \sigma}} \sum_{j=0}^{\infty}(a / b)^{j}<+\infty .
\end{aligned}
$$

Hence, $v_{n}(s)$ is well defined. Since

$$
\sum_{n=1}^{\infty}\left\|v_{n}(s)\right\|_{1} \leq k(s) \sum_{n=1}^{\infty} \frac{1}{\left(n-\frac{1}{2}\right)^{2 \sigma}} \sum_{j=0}^{\infty}(a / b)^{j}<+\infty,
$$

the Fredholm kernel $v(s)$ is also well defined.

Remark 2. We have $\widetilde{v}(s)=L(s)$ and $\widetilde{v}_{n}(s)=L_{n}(s)$ from the Taylor development. For the following theorem recall that there is a product on Fredholm kernels which is defined by

$$
(\lambda \otimes x) \cdot(\mu \otimes y)=\lambda(y) \mu \otimes x,
$$

for all $\lambda, \mu \in E^{*}$ and $x, y \in E$.

Theorem 3.3. (i) For every $s$ with $\operatorname{Re}(s)>\frac{1}{2}, v(s)$ is a Fredholm kernel of order 0 .

(ii) For every $n \geq 1$, the trace of $v^{n}(s)$ is given by

$$
\operatorname{Tr}\left(v^{n}(s)\right)=\sum_{a_{1}, \ldots, a_{n}} \frac{\left(\prod_{i=0}^{n-1} T^{i} x\right)^{2 s}}{1-(-1)^{n}\left(\prod_{i=0}^{n-1} T^{i} x\right)^{2}},
$$

with $x=x_{a_{1}, \ldots, a_{n}}=\left[0, \overline{a_{1}, \ldots, a_{n}}\right]$. The sum is extended over all $a_{1}, \ldots, a_{n} \in$ $\mathbb{N}-\{0\}$.

Proof. (i) $v(s)=\sum_{n=1}^{\infty} v_{n}(s)=\sum_{j=0}^{\infty} \mu_{j}(s) \otimes e_{j}$ with $\mu_{j}(s)=$ $\sum_{n=1}^{\infty} \lambda_{n, j}(s)$. We have

$$
\left\|\mu_{j}(s)\right\| \leq \sum_{n=1}^{\infty}\left\|\lambda_{n, j}(s)\right\| \leq \frac{k(s)}{b^{j}} \sum_{n=1}^{\infty} \frac{1}{\left(n-\frac{1}{2}\right)^{2 \sigma}} .
$$

Hence for all $\varepsilon>0, \sum_{j=0}^{\infty}\left\|\mu_{j}(s)\right\|^{\varepsilon}\left\|e_{j}\right\|^{\varepsilon}<\infty$. This proves (i).

(ii) Since $\sum_{n=1}^{\infty}\left\|v_{n}(s)\right\|_{1}<\infty$, we get for all $n \geq 1$

$$
v^{n}(s)=\sum_{a_{1}, \ldots, a_{n}} v_{a_{n}}(s) \ldots v_{a_{1}}(s) .
$$

Let $a_{1}, \ldots, a_{n}$ be fixed and put

$$
\begin{gathered}
R=T_{a_{1}} \circ \ldots \circ T_{a_{n}}, \\
S=\left(T_{a_{1}} \circ \ldots \circ T_{a_{n}}\right)^{2 s} \cdot\left(T_{a_{2}} \circ \ldots \circ T_{a_{n}}\right)^{2 s} \ldots\left(T_{a_{n}}\right)^{2 s}, \\
x=\left[0, \overline{a_{1}, \ldots, a_{n}}\right] .
\end{gathered}
$$


We recall that $T_{n}(z)=(z+n)^{-1}$. For $g \in E$ we have $L_{a_{n}}(s) \circ \ldots \circ$ $L_{a_{1}}(s)(g)=(g \circ R) \cdot S$. Let $\lambda \in \mathbb{C}$ be an eigenvalue of $L_{a_{n}}(s) \circ \ldots \circ L_{a_{1}}(s)$ and let $g \neq 0$ be an eigenfunction. For all $z \in D_{a}, g(R(z)) S(z)=\lambda g(z)$. Let $p \geq 0$ be the smallest integer such that $g^{(p)}(x) \neq 0$. Then the $p$ th derivative of the previous equality at $z=x$ yields (taking into account that $R(x)=x)$ :

$$
S(x)\left(R^{\prime}(x)\right)^{p} g^{(p)}(x)=\lambda g^{(p)}(x) \Rightarrow \lambda=\left(R^{\prime}(x)\right)^{p} S(x) .
$$

But we have

thus

$$
R(t)=\left[0, a_{1}, \ldots, a_{n}+t\right]=\frac{t p_{n-1}+p_{n}}{t q_{n-1}+q_{n}}
$$

$$
R^{\prime}(x)=\frac{(-1)^{n}}{\left(x q_{n-1}+q_{n}\right)^{2}},
$$

which implies $0<\left|R^{\prime}(x)\right|<1$. Thus the spectrum of $L_{a_{n}}(s) \circ \ldots \circ L_{a_{1}}(s)$ is contained in $\left\{\left(R^{\prime}(x)\right)^{p} S(x) ; p=0,1, \ldots\right\} \cup\{0\}$. We claim that there is equality. If $\lambda_{p}=\left(R^{\prime}(x)\right)^{p} S(x)$ is not an eigenvalue of the compact operator $L_{a_{n}}(s) \circ \ldots \circ L_{a_{1}}(s)$ there exists $h \in E$ such that

$$
h(R(z)) S(z)-\lambda_{p} h(z)=(z-x)^{p} \quad\left(\forall z \in D_{a}\right) .
$$

If $k \geq 0$ is the smallest integer such that $h^{(k)}(x) \neq 0$ we have

$$
h^{(k)}(x)\left(R^{\prime}(x)\right)^{k} S(x)-\lambda_{p} h^{(k)}(x)=p ! \delta_{k, p} .
$$

The two cases $k=p$ and $k \neq p$ both lead to a contradiction. We will now show that all the eigenvalues $\lambda_{p}=\left(R^{\prime}(x)\right)^{p} S(x)$ of $L_{a_{n}}(s) \circ \ldots \circ L_{a_{1}}(s)$ are simple (i.e. the associated spectral subspace is one-dimensional). We put

$$
\begin{gathered}
\theta=L_{a_{n}}(s) \circ \ldots \circ L_{a_{1}}(s), \\
E_{k}=\operatorname{Ker}\left(\theta-\lambda_{p} I\right)^{k} \quad(k \geq 1), \\
R_{k}=\underbrace{R \circ \ldots \circ R}_{k}, \quad S_{k}=\left(S \circ R_{k-1}\right)\left(S \circ R_{k-2}\right) \ldots S .
\end{gathered}
$$

If $f$ belongs to $E_{k}$ then

$$
\sum_{j=0}^{k} c_{j} \theta^{j}(f)=\sum_{j=0}^{k} c_{j}\left(f \circ R_{j}\right) S_{j}=0,
$$

where $c_{j}=\left(\begin{array}{c}k \\ j\end{array}\right)\left(-\lambda_{p}\right)^{k-j}$. By differentiating $m$ times the previous equality we obtain

$$
\begin{aligned}
0 & =\sum_{j=0}^{k} c_{j}\left(R^{\prime}(x)\right)^{m j} f^{(m)}(x)(S(x))^{j}+\ldots \\
& =f^{(m)}(x)\left[\left(R^{\prime}(x)\right)^{m} S(x)-\lambda_{p}\right]^{k}+\ldots
\end{aligned}
$$


where $+\ldots$ denotes a sum which depends only on $f(x), \ldots, f^{(m-1)}(x)$. We may deduce from this that $x$ is a zero of order $p$ for $f$ (if $f \neq 0$ ) and that if $g \neq 0$ is another function of $E_{k}$ then $g$ has the same derivatives at $x$ as $\left(g^{(p)}(x) / f^{(p)}(x)\right) f$, hence $g=\left(g^{(p)}(x) / f^{(p)}(x)\right) f$ and $\operatorname{dim} E_{k}=1$. We now finish the proof. From the above we have

$$
\begin{aligned}
\operatorname{Tr}\left(v_{a_{n}}(s) \ldots v_{a_{1}}(s)\right) & =\sum_{p=0}^{\infty} S(x)\left(R^{\prime}(x)\right)^{p} \\
& =\frac{S(x)}{1-R^{\prime}(x)}=\frac{\left(\prod_{i=0}^{n-1} T^{i} x\right)^{2 s}}{1-(-1)^{n}\left(\prod_{i=0}^{n-1} T^{i} x\right)^{2}} .
\end{aligned}
$$

Since $v^{n}(s)=\sum_{a_{1}, \ldots, a_{n}} v_{a_{n}}(s) \ldots v_{a_{1}}(s)$ we deduce (ii).

The following theorem gives some information on the localisation of eigenvalues of $L(s)$ and will be used later.

Proposition 3.4. (i) If $u>1$, there exists a number $R_{u}, 0 \leq R_{u}<1$, such that all eigenvalues of $L(s)$ for $\operatorname{Re}(s) \geq u$ have modulus $\leq R_{u}$.

(ii) If $s=1+i t$ with $t \neq 0$, then all eigenvalues of $L(s)$ have modulus $<1$.

(iii) 1 is the maximal eigenvalue of $L(1)$ (i.e. all other eigenvalues have modulus < 1).

Proof. (i) Suppose $\operatorname{Re}(s) \geq u$. Let $\lambda$ be an eigenvalue of $L(s)$ and let $f \neq 0$ be an associated eigenfunction. We have for all $0 \leq x \leq 1$

$$
\lambda f(x)=\sum_{n=1}^{\infty} f\left(\frac{1}{x+n}\right)\left(\frac{1}{x+n}\right)^{2 s} .
$$

Let $A$ be the maximum over $[0,1]$ for the function $(1+x)|f(x)|$. We have $|f(x)| \leq A(x+1)^{-1}$ on $[0,1]$, and there is $x_{0}$ such that $A=\left(1+x_{0}\right)\left|f\left(x_{0}\right)\right|$. Thus

$$
\begin{gathered}
|\lambda|\left|f\left(x_{0}\right)\right| \leq \sum_{n=1}^{\infty} \frac{A}{1+\frac{1}{x_{0}+n}}\left(\frac{1}{x_{0}+n}\right)^{2 u}, \\
|\lambda|\left|f\left(x_{0}\right)\right| \leq \frac{A}{1+\frac{1}{x_{0}+2}}\left[\left(\frac{1}{x_{0}+2}\right)^{2 u}-\left(\frac{1}{x_{0}+2}\right)^{2}\right] \\
+\sum_{n=1}^{\infty} \frac{A}{1+\frac{1}{x_{0}+n}}\left(\frac{1}{x_{0}+n}\right)^{2},
\end{gathered}
$$


since the last expression is equal to

$$
\sum_{\substack{n=1 \\ n \neq 2}}^{\infty} \frac{A}{1+\frac{1}{x_{0}+n}}\left(\frac{1}{x_{0}+n}\right)^{2}+\frac{A}{1+\frac{1}{x_{0}+2}}\left(\frac{1}{x_{0}+2}\right)^{2 u} .
$$

We have

$$
\left(\frac{1}{x_{0}+2}\right)^{2 u}-\left(\frac{1}{x_{0}+2}\right)^{2} \leq \operatorname{Max}_{0 \leq x \leq 1}\left[\left(\frac{1}{x+2}\right)^{2 u}-\left(\frac{1}{x+2}\right)^{2}\right]=\eta_{u}<0,
$$

hence,

$$
\begin{gathered}
|\lambda|\left|f\left(x_{0}\right)\right| \leq \frac{2}{3} A \eta_{u}+\frac{A}{x_{0}+1}, \\
|\lambda| \leq \frac{2}{3}\left(x_{0}+1\right) \eta_{u}+1 \leq \frac{2}{3} \eta_{u}+1=R_{u}<1 .
\end{gathered}
$$

(ii) The following lemma is elementary, the proof is left to the reader:

LEMMA 3.1. Let $\left(z_{n}\right)_{n \geq 1}$ be an absolutely convergent series of complex numbers such that $\left|\sum_{n=1}^{\infty} z_{n}\right|=\sum_{n=1}^{\infty}\left|z_{n}\right|$. Then there is an $\alpha \in \mathbb{C},|\alpha|=1$, such that $z_{n}=\alpha\left|z_{n}\right|$ for all $n \geq 1$.

Now let us prove (ii). Let $\lambda$ be an eigenvalue of $L(s)$ for $s=1+i t$, with $|\lambda| \geq 1$. We stick to the notations of (i). If one of the following two assertions:

$$
\begin{gathered}
|\lambda|\left|f\left(x_{0}\right)\right|<\sum_{n=1}^{\infty}\left|f\left(\frac{1}{x_{0}+n}\right)\right|\left(\frac{1}{x_{0}+n}\right)^{2}, \\
\left|f\left(\frac{1}{x_{0}+n}\right)\right|<\frac{A}{1+\frac{1}{x_{0}+n}} \quad \text { for at least one } n \geq 1
\end{gathered}
$$

holds then we will have

$$
|\lambda|\left|f\left(x_{0}\right)\right|<\sum_{n=1}^{\infty} \frac{A}{1+\frac{1}{x_{0}+n}}\left(\frac{1}{x_{0}+n}\right)^{2}=\left|f\left(x_{0}\right)\right|,
$$

which implies $|\lambda|<1$. Hence by using Lemma 3.1, there exists an $\alpha,|\alpha|=1$, so that, for every $n \geq 1$

$$
\begin{gathered}
\left(\frac{1}{x_{0}+n}\right)^{2 i t} f\left(\frac{1}{x_{0}+n}\right)=\alpha\left|f\left(\frac{1}{x_{0}+n}\right)\right| \\
\left|f\left(\frac{1}{x_{0}+n}\right)\right|=\frac{A}{1+\frac{1}{x_{0}+n}}
\end{gathered}
$$

The second equality shows that $|f(0)|=A>0$. Then the first one shows that the sequence $\left(x_{0}+n\right)^{-2 i t}$ converges but this is impossible if $t \neq 0$. 
(iii) 1 is an eigenvalue of $L(1)$ and $(z+1)^{-1}$ is an associated eigenfunction. Let $\lambda \in \mathbb{C}$ be an eigenvalue of $L(1)$ with $|\lambda| \geq 1$ and $f$ an eigenfunction. The proof of (ii) with the same notations shows that for all $n \geq 1$

$$
f\left(\frac{1}{x_{0}+n}\right)=\frac{\alpha A}{1+\frac{1}{x_{0}+n}} .
$$

Since $f$ is analytic we deduce $f(z)=\alpha A(1+z)^{-1}$ and finally $\lambda=1$, which proves (iii).

Proposition 3.5. The map $s \mapsto v(s)$ is holomorphic for $\operatorname{Re}(s)>\frac{1}{2}$.

Proof. Let $z \in \mathbb{C}$ be given such that $|z-1|=b$. We define $\psi_{n}(s)=$ $(z+n)^{-2 s}$ for $\operatorname{Re}(s)>\frac{1}{2}$. One has

$$
\begin{gathered}
\left|\psi_{n}(s+h)-\psi_{n}(s)\right| \leq|h| \sup _{[s, s+h]}\left|\psi_{n}^{\prime}(u)\right|, \\
\left|\psi_{n}(s+h)-\psi_{n}(s)-h \psi_{n}^{\prime}(s)\right| \leq\left(|h|^{2} / 2\right) \sup _{[s, s+h]}\left|\psi_{n}^{\prime \prime}(u)\right|,
\end{gathered}
$$

where $[s, s+h]$ denotes the segment in $\mathbb{C}$ with endpoints $s$ and $s+h$. Put $u=s+t h, 0 \leq t \leq 1,|h| \leq c$ where $c$ will be specified later. Then

$$
\left|\psi_{n}^{\prime}(u)\right| \leq 2(\log (n+1+b)+\pi) \frac{1}{|n+z|^{\operatorname{Re}(2 s+2 t h)}} e^{-\operatorname{Im}(2 s+2 t h) \operatorname{Arg}(1 /(z+n))} .
$$

The argument of the exponential function is dominated by $2 \pi(|s|+c)=$ $M(s)$ and furthermore $|n+z|^{-\operatorname{Re}(2 t h)} \leq(n+1+b)^{2 c}$, hence

$$
\sup _{[s, s+h]}\left|\psi^{\prime}(u)\right| \leq 2 \frac{e^{M(s)}}{\left(n-\frac{1}{2}\right)^{2 \sigma}}(n+1+b)^{2 c}(\log (n+1+b)+\pi)=b_{n}(s, c) .
$$

Similarly:

$$
\sup _{[s, s+h]}\left|\psi^{\prime \prime}(u)\right| \leq 4 \frac{e^{M(s)}}{\left(n-\frac{1}{2}\right)^{2 \sigma}}(n+1+b)^{2 c}(\log (n+1+b)+\pi)^{2}=c_{n}(s, c) .
$$

By Cauchy's inequalities we get

$$
\begin{gathered}
\left\|\lambda_{n, j}(s+h)-\lambda_{n, j}(s)-h \theta_{n, j}(s)\right\| \leq \frac{c_{n}(s, c)}{b^{j}} \frac{|h|^{2}}{2}, \\
\left\|\lambda_{n, j}(s+h)-\lambda_{n, j}(s)\right\| \leq \frac{b_{n}(s, c)}{b^{j}}|h|,
\end{gathered}
$$

where $\theta_{n, j}(s) \in E^{*}$ is given by $\theta_{n, j}(s)(f)=(j !)^{-1} h_{n}^{(j)}(1)$ and $h_{n}$ by

$$
h_{n}(z)=2 \log \left(\frac{1}{z+n}\right) f\left(\frac{1}{z+n}\right)\left(\frac{1}{z+n}\right)^{2 s} .
$$


Hence $\lambda_{n, j}(s)$ is holomorphic and $\lambda_{n, j}^{\prime}(s)\left(=\theta_{n, j}(s)\right)$ satisfies

$$
\left\|\lambda_{n, j}^{\prime}(s)\right\| \leq \frac{b_{n}(s, c)}{b^{j}} .
$$

Thus $\sum_{j=0}^{\infty} \lambda_{n, j}^{\prime}(s) \otimes e_{j}$ is uniformly convergent on every bounded subset of $\operatorname{Re}(s)>\frac{1}{2}$ since we may bound $M(s)$. Hence $v_{n}(s)$ is holomorphic and $v_{n}^{\prime}(s)=\sum_{j=0}^{\infty} \lambda_{n, j}^{\prime}(s) \otimes e_{j}$. Moreover, we get $\left\|v_{n}^{\prime}(s)\right\|_{1} \leq \sum_{j=0}^{\infty}\left\|\lambda_{j}^{\prime}(s)\right\|\left\|e_{j}\right\|$ $\leq b_{n}(s, c) \sum_{j=0}^{\infty}(a / b)^{j}$. The series $\sum_{n=1}^{\infty} v_{n}^{\prime}(s)$ is also uniformly convergent on every bounded subset $K$ of $\operatorname{Re}(s)>\frac{1}{2}$ since we may bound $M(s)$ uniformly and choose constants $c, c^{\prime}$ such that $2 \sigma-2 c>c^{\prime}>1$ for all $s$ in $K$. Thus $v(s)$ is holomorphic and $v^{\prime}(s)=\sum_{n=1}^{\infty} v_{n}^{\prime}(s)$.

Now we claim that 1 is a simple eigenvalue of $L(1)$. In fact, we will establish a stronger result. Let $P: L^{1} \rightarrow L^{1}$ (where $L^{1}=L^{1}[0,1]$ ) be the operator defined by

$$
P(f)(x)=\sum_{n=1}^{\infty} f\left(\frac{1}{x+n}\right)\left(\frac{1}{x+n}\right)^{2} .
$$

$P$ is the Perron-Frobenius operator associated to the continued fraction transformation $T$, that is, for all $g \in L^{\infty}$ and $f \in L^{1}$

$$
\int_{0}^{1}(g \circ T) \cdot f d x=\int_{0}^{1} g \cdot P(f) d x .
$$

1 is an eigenvalue of $P$, an eigenfunction being $f_{0}(x)=1 / \log 2(1+x)$. The following theorem specifies this statement.

TheOREM 3.4. The operator $P$ admits 1 as a simple eigenvalue. More precisely, the spectral subspace associated to 1 is generated by $f_{0}$.

Proof. Let $f \in L^{1}$ such that $P(f)=f$. For all $g \in L^{\infty}$ and $k=$ $0,1,2, \ldots$, we have

$$
\int_{0}^{1} g \circ T^{k} f d x=\int_{0}^{1} g f d x .
$$

We put $h(x)=f(x)(1+x) \log 2$. We can rewrite the previous equality as

$$
\int_{0}^{1} g \circ T^{k} h d \mu=\int_{0}^{1} g h d \mu .
$$

We can deduce from this by using the strongly mixing property of $T$ that

$$
f=\left(\int_{0}^{1} f d x\right) f_{0} \quad \text { a.e. }
$$


The proof will be complete if we show that $\operatorname{Ker}(P-I)=\operatorname{Ker}(P-I)^{2}$. Let $f \in \operatorname{Ker}(P-I)^{2}$. There is a complex number $\alpha$ such that $(P-I) f=\alpha f_{0}$. After integration over $[0,1]$ we obtain $\alpha=0$ since $\int_{0}^{1} \operatorname{Pf} d x=\int_{0}^{1} f d x$. Hence $P f=f$ and this concludes the proof.

Corollary 3.2. The operator L(1) admits 1 as a simple eigenvalue.

By part (iii) of Proposition 3.4, there exists a number $R, 0<R<1$, such that all the eigenvalues of $L(1)$ different from 1 are contained in the open disc $D(0, R)$. Let $W$ be the complement of $D(0, R)$. From Corollary 3.2, perturbation theory (see [2], p. 587) and the fact that $L(s)$ is holomorphic (since $v(s)$ is holomorphic by Proposition 3.5) there exists $\delta, 0<\delta<\frac{1}{2}$, and a holomorphic function $s \mapsto \theta(s) \in \mathbb{C}$ defined for $|s-1|<\delta$, such that $\operatorname{Sp}(L(s)) \cap W=\{\theta(s)\}$ (Sp = spectrum). Thus:

Corollary 3.3. For $|s-1|<\delta, \theta(s)$ is the eigenvalue of maximal modulus for $L(s)$.

We now introduce

$$
\begin{array}{ll}
f_{1}(z, s)=\operatorname{det}(I-z v(s)), & F_{1}(z, s)=f_{1}(z, s) f_{1}(-z, s), \\
f_{2}(z, s)=\operatorname{det}(I-z v(s+1)), & F_{2}(z, s)=f_{2}(z, s) f_{2}(-z, s) .
\end{array}
$$

From the fact that $v \mapsto \operatorname{det}(I+v)$ is analytic [6] and $s \mapsto v(s)$ is holomorphic, $f_{1}, f_{2}, F_{1}, F_{2}$ are holomorphic functions for $z \in \mathbb{C}$ and $\operatorname{Re}(s)>\frac{1}{2}$.

TheOrem 3.5. For $\operatorname{Re}(s)>1$ we have

$$
F(s)=\frac{-1}{2} \frac{\partial F_{1} / \partial z(1, s)}{F_{1}(1, s)}+G(s),
$$

where $G$ is a holomorphic function for $\operatorname{Re}(s)>\frac{1}{2}$.

Remark. If $\operatorname{Re}(s)>1$, all the eigenvalues of $L(s)$ have modulus $<1$ from Proposition 3.4, hence $F_{1}(1, s) \neq 0$.

Proof. According to Fredholm's theory [6] we have

$$
\operatorname{det}(I-z v(s))=\exp \left(-\sum_{n=1}^{\infty} \frac{z^{n}}{n} \operatorname{Tr}\left(v^{n}(s)\right)\right) \text {. }
$$

This equality holds for $|z|$ sufficiently small (in fact for $|z|<\|v(s)\|_{1}^{-1}$ ).

We put for $n \geq 1$ :

$$
A_{n}(s)=\sum_{a_{1}, \ldots, a_{n}}\left(x T x \ldots T^{n-1} x\right)^{2 \alpha_{n} s},
$$

where as usual $x=x_{a_{1}, \ldots, a_{n}}=\left[0, \overline{a_{1}, \ldots, a_{n}}\right]$. The series

$$
F(s)=\sum_{n=1}^{\infty} A_{n}(s)=\sum_{n=1}^{\infty} \sum_{a_{1}, \ldots, a_{n}}\left(x T x \ldots T^{n-1} x\right)^{2 \alpha_{n} s}
$$


is absolutely convergent for $\operatorname{Re}(s)>1$. Theorem 3.3 and relation $(*)$ show by analytic continuation that

$$
F_{2}(z, s)=\exp \left(2 \sum_{n \text { even }} \frac{z^{n}}{n} A_{n}(s)\right) F_{1}(z, s),
$$

for $|z|<1$ and $\operatorname{Re}(s)>1$. Thus

$$
2 \sum_{n \text { even }} A_{n}(s)=\frac{1}{F_{2}(1, s)} \frac{\partial F_{2}}{\partial z}(1, s)-\frac{1}{F_{1}(1, s)} \frac{\partial F_{1}}{\partial z}(1, s),
$$

for $\operatorname{Re}(s)>1$. Notice that $F_{2}(1, s) \neq 0$ if $\operatorname{Re}(s)>\frac{1}{2}$. We now set for $z \in \mathbb{C}$ and $\operatorname{Re}(s)>\frac{1}{2}$

$$
F_{3}(z, s)=\frac{f_{2}(-z, 2 s)}{f_{2}(z, 2 s)}, \quad F_{4}(z, s)=\frac{f_{1}(z, 2 s)}{f_{1}(-z, 2 s)} .
$$

Let $\frac{1}{2}<d<1$. From Proposition 3.4, $F_{3}, F_{4}$ are holomorphic and nonvanishing for $|z|<\left(R_{2 d}\right)^{-1}$ and $\operatorname{Re}(s)>d$ (with the notations of this proposition). With the previous argument we have for $\operatorname{Re}(s)>1$

$$
2 \sum_{n \text { odd }} A_{n}(s)=\frac{\partial F_{3} / \partial z(1, s)}{F_{3}(1, s)}-\frac{\partial F_{4} / \partial z(1, s)}{F_{4}(1, s)} .
$$

The right member of this equality is holomorphic for $\operatorname{Re}(s)>d$. Since $\frac{1}{2}<d<1$ is arbitrary we see that the left member can be extended holomorphically to $\operatorname{Re}(s)>\frac{1}{2}$. Since

$$
F(s)=\sum_{n \text { even }} A_{n}(s)+\sum_{n \text { odd }} A_{n}(s),
$$

the theorem is proved.

Corollary 3.4. (i) F extends meromorphically to $\operatorname{Re}(s)>\frac{1}{2}$. Moreover, on the line $\operatorname{Re}(s)=1, F$ has only one pole which is simple and located at $s=1$.

(ii) The residue is equal to $-1 / 2 \theta^{\prime}(1)$ (we will see later that $\theta^{\prime}(1) \neq 0$ ).

(iii) $\pi_{2}(X)=o\left(e^{X}\right)$.

Proof. The first part of (i) is evident from Theorem 3.5. Put $s=1+i t$ with $t \neq 0$. From Proposition 3.4 all eigenvalues of $L(s)$ have modulus $<1$, which implies $F_{1}(1, s) \neq 0$. Hence $s$ is not a pole for $F$. We show now that 1 is a simple pole. Let $\left|\theta_{1}\right| \geq\left|\theta_{2}\right| \geq \ldots$ be the eigenvalues (with $\theta(s)=\theta_{1}$ ) of $L(s)$ for $|s-1|<\delta$, each counted according to its multiplicity. Since $v(s)$ is of order 0 (Theorem 3.3) we have [5]

$$
\sum_{i=1}^{\infty}\left|\theta_{i}\right|<\infty \quad \text { and } \quad f_{1}(z, s)=\prod_{i \geq 1}\left(1-z \theta_{i}\right) .
$$


By putting $G(z, s)=\prod_{i>2}\left(1-z^{2} \theta_{i}^{2}\right)$ we can write $F_{1}(z, s)=\left(1-z^{2} \theta^{2}(s)\right)$ $\times G(z, s)$ and the function $G$ is defined for $|s-1|<\delta$ and $z \in \mathbb{C}$. Furthermore, if $s$ is fixed the map $z \mapsto G(z, s)(z \in \mathbb{C})$ is holomorphic. Take $\delta^{\prime}<\delta$ such that $\theta(s)$ remains bounded if $|s-1|<\delta^{\prime}$. Then there is an $\varepsilon>0$ such that for $|z|<\varepsilon$ and $|s-1|<\delta^{\prime}, 1-z^{2} \theta^{2}(s) \neq 0$. Hence $G$ is holomorphic in this open set. It follows from Levi's Theorem $([18]$, p. 8$)$ that $G$ is in fact holomorphic for $z \in \mathbb{C}$ and $|s-1|<\delta^{\prime}$. Hence

$$
\frac{\partial F_{1}}{\partial z}(1,1)=-2 G(1,1), \quad \frac{\partial F_{1}}{\partial s}(1,1)=-2 \theta^{\prime}(1) G(1,1) .
$$

But

$$
\begin{aligned}
\frac{\partial F_{1}}{\partial z}(1,1) & =\frac{\partial f_{1}}{\partial z}(1,1) f_{1}(-1,1)-f_{1}(1,1) \frac{\partial f_{1}}{\partial z}(-1,1) \\
& =\frac{\partial f_{1}}{\partial z}(1,1) f_{1}(-1,1) .
\end{aligned}
$$

Since 1 is a simple eigenvalue of $L(1)$ of maximal modulus we have $\partial f_{1} / \partial z(1,1) \neq 0$ and $f_{1}(-1,1) \neq 0$. It follows that $\partial F_{1} / \partial z(1,1) \neq 0$ and $\partial F_{1} / \partial s(1,1) \neq 0$. This proves (ii).

For (iii), we have shown in the previous theorem that the function $\sum_{n \text { odd }} A_{n}(s)$, which is holomorphic for $\operatorname{Re}(s)>1$, can be extended to $\operatorname{Re}(s)>\frac{1}{2}$. From Ikehara's tauberian theorem we get immediately

$$
\sum_{\substack{\varrho(m) \leq X \\|m| \text { odd }}} 1=o\left(e^{X}\right) .
$$

Thus $\pi_{2}(X)=o\left(e^{X}\right)$ and this completes the proof of the corollary.

Proposition 3.6. If $|s-1|<\delta$ with s real we have $\theta(s)>0$.

Proof. Fix $s$ and denote by $k$ the order of multiplicity of the eigenvalue $\theta(s)$. We have for all $n \geq 1$

$$
\operatorname{Tr}\left(v^{n}(s)\right)=\sum_{i=1}^{\infty} \theta_{i}^{n},
$$

where as in the proof of the previous corollary $\left|\theta_{1}\right| \geq\left|\theta_{2}\right| \geq \ldots$ are the eigenvalues of $L(s)$. Since $\sum_{i=1}^{\infty}\left|\theta_{i}\right|<+\infty$ (because $v(s)$ is of order 0 from Theorem 3.3) and $\left(\theta_{i} / \theta(s)\right)^{n} \rightarrow 0$ for $\theta_{i} \neq \theta(s)$ and $n \rightarrow \infty$ we obtain

$$
\lim _{n \rightarrow \infty} \operatorname{Tr}\left(v^{n}(s)\right) / \theta^{n}(s)=k .
$$

This implies $\theta(s)>0$ since if $s$ is real $\operatorname{Tr}\left(v^{n}(s)\right)>0$ for all $n \geq 1$ from Theorem 3.3.

The following inequality is fundamental. It combines $\theta(s)$ and the ergodic properties of $T$, the transformation of continued fractions. This inequality 
will allow us to explicitly compute $\theta^{\prime}(1)$ and thus the residue of $F(s)$ at $s=1$.

TheOREm 3.6. For $|s-1|<\delta$ with $s$ real we have $\log \theta(s) \geq(1-s) h_{\mu}(T)$ where $h_{\mu}(T)$ is the entropy of $T$ with respect to Gauss's measure $\mu$.

Pro of. Let $f \in E$. We have

$$
\begin{aligned}
L^{n}(s)(f) & =\sum_{a_{1}, \ldots, a_{n}}\left(f \circ T_{a_{1}} \circ \ldots \circ T_{a_{n}}\right)\left(T_{a_{1}} \circ \ldots \circ T_{a_{n}}\right)^{2 s} \ldots\left(T_{a_{n}}\right)^{2 s}, \\
\left\|L^{n}(s)\right\| & \geq \operatorname{Max}_{D_{a}}\left|\sum_{a_{1}, \ldots, a_{n}}\left(T_{a_{1}} \circ \ldots \circ T_{a_{n}}\right)^{2 s} \ldots\left(T_{a_{n}}\right)^{2 s}\right| \\
& \geq \operatorname{Max}_{[0,1]} \sum_{a_{1}, \ldots, a_{n}}\left(T_{a_{1}} \circ \ldots \circ T_{a_{n}}\right)^{2 s} \ldots\left(T_{a_{n}}\right)^{2 s} \quad(s \in \mathbb{R}) .
\end{aligned}
$$

For $x \in[0,1]\left(T_{a_{1}} \circ \ldots \circ T_{a_{n}}\right)(x) \ldots T_{a_{n}}(x)=y T y \ldots T^{n-1} y$, where $y$ is given by

$$
y=\left[0, a_{1}, \ldots, a_{n}+x\right]=\frac{x p_{n-1}+p_{n}}{x q_{n-1}+q_{n}} .
$$

Hence from Lemma 2.1

$$
y T y \ldots T^{n-1} y=\frac{1}{x q_{n-1}+q_{n}} .
$$

Thus

$$
\operatorname{Max}_{[0,1]} \sum_{a_{1}, \ldots, a_{n}}\left(T_{a_{1}} \circ \ldots \circ T_{a_{n}}\right)^{2 s} \ldots\left(T_{a_{n}}\right)^{2 s}=\sum_{a_{1}, \ldots, a_{n}} \frac{1}{q_{n}^{2 s}} .
$$

From the previous equality, the spectral radius formula and Proposition 3.6 we obtain

$$
\log \theta(s)=\lim _{n \rightarrow \infty} \frac{1}{n} \log \left\|L^{n}(s)\right\| \geq \limsup _{n \rightarrow \infty} \frac{1}{n} \log \sum_{a_{1}, \ldots, a_{n}} \frac{1}{q_{n}^{2 s}} .
$$

Let $I_{a_{1}, \ldots, a_{n}}$ be the fundamental interval corresponding to $a_{1}, \ldots, a_{n}$. Since the fundamental intervals generate the Borel subsets of $[0,1]$ we deduce from the Kolmogorov-Sinai Theorem

$$
(s-1) h_{\mu}(T)=\lim _{n \rightarrow \infty} \frac{-1}{n} \sum_{a_{1}, \ldots, a_{n}} \mu\left(I_{a_{1}, \ldots, a_{n}}\right) \log \mu\left(I_{a_{1}, \ldots, a_{n}}\right)^{s-1} .
$$

From the convexity of the function - log we get

$$
(1-s) h_{\mu}(T) \leq \liminf _{n \rightarrow \infty} \frac{1}{n} \log \sum_{a_{1}, \ldots, a_{n}} \mu\left(I_{a_{1}, \ldots, a_{n}}\right)^{s} .
$$

If $m$ denotes the Lebesgue measure, the inequality $\mu(A) \leq(\log 2)^{-1} m(A)$ 
valid for all Borel subsets $A$ of $[0,1]$ leads to

$$
\begin{aligned}
(1-s) h_{\mu}(T) & \leq \liminf _{n \rightarrow \infty} \frac{1}{n} \log \sum_{a_{1}, \ldots, a_{n}} m\left(I_{a_{1}, \ldots, a_{n}}\right)^{s} \\
& \leq \liminf _{n \rightarrow \infty} \frac{1}{n} \log \sum_{a_{1}, \ldots, a_{n}}\left(\frac{1}{q_{n}\left(q_{n}+q_{n-1}\right)}\right)^{s} \\
& \leq \liminf _{n \rightarrow \infty} \frac{1}{n} \log \sum_{a_{1}, \ldots, a_{n}} \frac{1}{q_{n}^{2 s}} \leq \log \theta(s) .
\end{aligned}
$$

This proves the desired inequality.

The function $\log \theta(s)-(1-s) h_{\mu}(T)$ is $\geq 0$ for $1-\delta<s<1+\delta$ and vanishes at $s=1$, thus its derivative is zero at $s=1$ and we get $\theta^{\prime}(1)=-h_{\mu}(T)$. Hence applying Ikehara's theorem for the function $F(s)=$ $\int_{0}^{\infty} e^{-s t} d \pi_{1}(t)$ we obtain from Corollary 3.4

$$
\sum_{\varrho(m) \leq X} 1 \sim \frac{3 \log 2}{\pi^{2}} e^{X} \quad(X \rightarrow \infty) .
$$

From Proposition 3.3 and $\pi_{2}(X)=o\left(e^{X}\right)$ (Corollary 3.4(iii)) we get the desired theorem:

\section{THEOREM II.}

$$
\sum_{\varrho(x) \leq X} 1 \sim \frac{3 \log 2}{\pi^{2}} e^{X} \quad(X \rightarrow+\infty) .
$$

Notice that in this theorem we can restrict ourselves to the reduced quadratic numbers $x$ such that $\operatorname{per}(x)$ is even.

\section{References}

[1] P. Billingsley, Ergodic Theory and Information, Wiley, New York 1965.

[2] N. Dunford and J. Schwartz, Linear Operators, Part I, Wiley-Interscience, 1963.

[3] C. Faivre, Distribution des constantes de Lévy des nombres quadratiques, Ph.D. Thesis, Université de Provence, 1990.

[4] E. Galois, Démonstration d'un théorème sur les fractions continues périodiques, Ann. Math. Pures Appl. 19 (1828-1829), 294-301.

[5] A. Grothendieck, Produits tensoriels topologiques et espaces nucléaires, Mem. Amer. Math. Soc. 16 (1955).

[6] —, La théorie de Fredholm, Bull. Soc. Math. France 84 (1956), 319-384.

[7] G. H. Hardy and E. M. Wright, An Introduction to the Theory of Numbers, Clarendon Press, Oxford 1968.

[8] D. Hejhal, The Selberg Trace Formula for $\operatorname{PSL}(2, \mathbb{R})$, Vol. 2, Lecture Notes in Math. 1001, Springer, 1983.

[9] H. Jager et P. Liardet, Distributions arithmétiques des dénominateurs des convergents de fractions continues, Indag. Math. 50 (1988), 181-197. 
[10] N. V. Kuznetsov, The distribution of norms of primitive hyperbolic classes of the modular group, Soviet Math. Dokl. 19 (5) (1978), 1053-1056.

[11] E. Landau, Elementary Number Theory, Chelsea, New York 1958.

[12] P. Lévy, Sur les lois de probabilités dont dépendent les quotients complets et incomplets d'une fraction continue, Bull. Soc. Math. France 57 (1929), 178-194.

[13] D. Mayer, On a $\zeta$ function related to the continued fraction transformation, Bull. Soc. Math. France 104 (1976), 195-203.

[14] M. Pollicott, Distribution of closed geodesics on the modular surface and quadratic irrationals, Bull. Soc. Math. France 144 (1986), 431-446.

[15] D. Ruelle, Zeta-functions for expanding maps and Anosov flows, Invent. Math. 34 (1976), 231-242.

[16] P. Sarnak, Class numbers of indefinite binary quadratic forms, J. Number Theory 15 (1982), 229-247.

[17] G. Shimura, Introduction to the Arithmetic Theory of Automorphic Functions, Publ. Math. Soc. Japan 11, Iwanami Shoten and Princeton University Press, 1971.

[18] Y.-T. Siu, Techniques of Extension of Analytic Objects, Lectures Notes in Pure and Appl. Math. 8, Dekker, 1974.

[19] H. Smith, Note on the theory of the Pellian equation, and of binary quadratic forms of a positive determinant, Proc. London Math. Soc. 7 (1876), 199-208.

URA 225

UNIVERSITÉ DE PROVENCE, U.F.R.-M.I.M.

3, PLACE VICTOR HUGO

13331 MARSEILLE CEDEX 3, FRANCE

Received on 10.7 .1990

and in revised form on 5.6.1991 